\section{Leben des Benvenuto Cellini}

G.s erster Kontakt mit der Lebensbeschreibung des Florentiner Goldschmieds und Bildhauers Benvenuto Cellini datiert in den Sommer des Jahres 1795 und steht einerseits im Kontext der Planungen für eine erneute Italienreise, andererseits im Umfeld eines großangelegten, nie realisierten Publikationsprojektes: Zusammen mit Johann Heinrich Meyer plante G. eine umfassende kunsthistorische und kulturmorphologische Enzyklopädie über Italien und die dortigen Verhältnisse in Einzeldarstellungen. Als Materialbasis hierfür dienten G. die geläufigen Sammlungen von Künstlerviten. Ob es in diesem Zusammenhang eher die Äußerung Giorgio Vasaris über seinen Künstlerkollegen in seinen Vite (1568) war, die G. zu Cellini führte, oder vielmehr der Kontakt zu Charles Gore, der sich seit 1791 in Weimar aufhielt und G. im Sommer 1795 sein Exemplar der Cellini-Vita auslieh, ist nicht eindeutig zu klären. Jedenfalls lässt sich G. im Dezember 1795 von Georg Christoph Lichtenberg ein Exemplar von Cellinis Due Trattati, uno intorno alle otto principali arti dell'oreficeria. L'altro in materia dell'arte della scultura; dove si veggono infiniti segreti nel lavorar le figure di marmo, \& nel gettarle di bronzo (Erstausgabe Florenz 1568) aus der Göttinger Bibliothek beschaffen. Am 17. August des Folgejahres schickt er eine Übersicht seiner Beiträge für die Horen an Schiller, unter denen für die November- und Dezemberausgabe auch Teile aus der Übersetzung von Cellinis Vita angekündigt werden. Parallel zur Fertigstellung des Wilhelm Meister arbeitet G. von Januar 1796 bis Juni 1797 an seiner Übersetzung, die zu diesem Zeitpunkt noch nicht den vollständigen Text der Vita umfasst. Die einzelnen Stücke erscheinen mit verbindenden Zwischentexten sukzessive in den Horen, und zwar in den Jahrgängen 1796 (4.-7. und 9.11. Stück) und 1797 (1.-4. und 6. Stück). 1798 kommt bei J. Bauer in Braunschweig ein Raubdruck dieses Textbestandes heraus. In dieser Zeit plant G., dem kurz danach aufgegebenen Projekt der Italien-Enzyklopädie die CelliniÜbersetzung als Schlussband anzugliedern. Nach längeren Verhandlungen mit Cotta unterstützt dieser schließlich die Publikation einer von G. überarbeiteten und durch einen kunst- und kulturhistorischen Anhang ergänzten Gesamtübersetzung von Cellinis Vita, die dann zur Ostermesse im April 1803 in einer Auflage von tausend Exemplaren erscheint.

G.s Übersetzung basiert nicht, wie bisweilen in der Literatur gemutmaßt, auf der ersten englischen, Sir Joshua Reynolds gewidmeten Übersetzung der Cellinischen Vita von Thomas $\mathrm{Nu}$ gent aus dem Jahr 1771. Hieraus übernimmt G. lediglich die Buch- und Kapiteleinteilung sowie die erläuternden Zwischentitel. Textgrundlage war auch nicht das heute in der Biblioteca Laurenziana verwahrte Originalmanuskript, das erst 1829 von Francesco Tassi wiederentdeckt und publiziert wurde, sondern eine teilweise fehlerhafte, geglättete, gekürzte und der italienischen Schriftsprache angeglichene, heute verlorene Abschrift des Originals, die Antonio Cocchi 1728 in Neapel unter Angabe des fingierten Druckorts und Druckers »In Colonia. Per Pietro Martello« herausgegeben hatte: Vita di Benvenuto Cellini orefice e Scultore Fiorentino, da lui medesimo scritta, Nella quale molte curiose particolarità si toccano appartenenti alle Arti ed all'Istoria del suo tempo, tratte da un'ottimo manoscritto, e Dedicato All'Eccelenza Di Mylord Boyle.

Die Frage nach der Qualität der G.schen Übersetzung wurde seit den ersten Rezensionen des Werkes ebenso heftig wie kontrovers diskutiert. Hatte Ludwig Ferdinand Huber in seiner Besprechung vom 24.6.1803 im Freimütigen noch die »täuschende Treue«, mit welcher die Übersetzung »die naivsten Eigentümlichkeiten einer fremden Nation in einem entlegenen Zeitalter ausdrückt « (zitiert nach MA 7, S. 748), gelobt, so erkannte bereits Karl Ludwig Fernow am 11.1.1804 in der Jenaischen Allgemeinen Literatur-Zeitung reinigende und veredelnde Eingriffe G.s in den stellenweise korrupten Ursprungstext: "G o e the s Übersetzung kann uns für ein Original gelten; denn eben das, was die eigenen Werke dieses Dichters vor allen auszeichnet, die seltene Gabe, sich immer so in seinen Gegenstand zu verwandeln, daß seine eigene Individualität aus der Darstellung verschwindet, und jene des Objekts in seiner eigentümlichen Gestalt und Farbe um so reiner her- 
vortritt, ist auch ein schätzbarer Vorzug dieser Übersetzung. Wer der italienischen Sprache hinreichend kundig ist, wird nicht nur das Eigentümliche der Schreibart Cellinis, seine völlig kunstlose, gerade, nicht selten derbe, zugleich aber auch zierliche, oft anmutige Diktion in der Goetheschen Übersetzung wiederfinden, sondern auch bei einer näheren Vergleichung bemerken, daß diese letztere, neben aller Treue in Rücksicht auf Inhalt und Ausdruck, eine gewisse Reinheit und Veredelung zeigt, die der Diktion des Originals zur Schönheit noch mangelt« (zitiert nach ebd., S. 750)

Trotz seiner Neigung zur Idealisierung und trotz seiner Goethe-Verehrung forderte dann Karl Vossler in seiner berühmten Philippika (zuerst 1900 in der Beilage zur Münchener Allgemeinen Zeitung, dann erneut 1903 in der Einleitung zu dem von ihm herausgegebenen Cellini-Band der Heinemannschen Werkausgabe G.s) aus der Perspektive des Romanisten stärkere Authentizität in der Übertragung der unmittelbaren, ungefilterten, emotional hoch aufgeladenen Sprache Cellinis. Seine Kritik kulminiert in einer Fragenkaskade mit polemischem Unterton: „Wo bleibt die unerhörte sinnliche Kraft und Plastik, wo das Temperament, die Leidenschaft, die Rohheit, die Verwirrtheit, die Hast und der Schwulst? Und ganz besonders: wo jener bizarre Zwiespalt von naiv und rhetorisch, von natürlich und gesucht, von geschwätzig und schlagend, von volkstümlich und manieriert, der für den emporgekommenen Sohn des Volkes so außerordentlich charakteristisch ist? « (Vossler, S. 11). Doch trotz G.s mangelhaften Kenntnissen der italienischen Sprache, die Vossler ihm unterstellt, und trotz der allzu freien Übertragung setze sich Cellinis "so urkräftiger, so urbizarrer, so leidenschaftlicher und kühner" Stil (ebd.) im Text einigermaBen durch. Und da es sich um den Titan der deutschen Dichtung handelt, der hier vor Gericht steht, setzt Vossler schließlich doch zu einer Ehrenrettung an, indem er einerseits G.s Kunst, »die immer nur in stille Höhen strebt« (ebd., S. 12), als inkompatibel mit dem eruptiven Charakter Cellinis charakterisiert und andererseits die Assimilation dieses herausragenden italienischen Kulturguts an den deutschen Geist als bleibendes Verdienst G.s hervorhebt.
Irmgard Nickel, Erwin Koppen und Marianne Bockelkamp haben in der Folge mit je unterschiedlichen Akzenten G.s Leistung in detaillierten philologischen Untersuchungen zu würdigen versucht. Die jeweiligen Argumente in dieser Qualitätsdebatte richteten sich nach den Anforderungen, die die Kritiker an eine Übersetzung stellten: Erwartete man eine möglichst textnahe und stilgetreue Wiedergabe des Originals oder schloss man sich eher G.s eigenen späteren übersetzungstheoretischen Überlegungen an, wie er sie beispielsweise in einem Brief an Thomas Carlyle vom 20.7.1827 formulierte, wo er den Übersetzer als »Vermittler dieses allgemein geistigen Handels « des der ganzen Menschheit als Tauschware Angehörenden bezeichnet, Übersetzen somit als eine Art von Kulturtransfer und Dolmetscherleistung im Warentausch definiert hatte. In vergleichbarem Sinne schrieb er am 7.9.1821 an Joseph Stanislaus Zauper speziell über seine Cellini-Übersetzung: "Wegen Cellini und Rameau sage gleichfalls Dank; ich habe diese beiden seltsamen Figuren herübergeführt, damit man das Fremdeste im vaterländischen Kreis gewahr werde. Liest man dergleichen Darstellungen im Original, so sehen sie ganz anders aus und nöthigen uns, um sie nur einigermaßen zu genießen und zu nützen, in ganz fremde Kreise; bey Übersetzungen aber sind wir gefördert, wie auf einer Handelsmesse, wo uns der Entfernteste seine Ware herbeybringt. In beiden Fällen habe [ich] dem Bedürfnis nachzuhelfen gesucht.« Norbert Miller schließlich hat in seiner Einleitung zum Kommentar der Münchner Ausgabe einen salomonischen Ausgleich zwischen diesen streitenden Parteien gesucht, indem er sich würdigend-vermittelnd in die Debatte einschaltete und die Argumentation damit auf eine neue Stufe hob: "Das Außerordentliche, das schlechthin Unvergleichliche des Benvenuto Cellini wird in Goethes Übersetzungswerk gerade darum erkennbar, weil dieser seine Weltwahrnehmung zur camera obscura für die Sehweise seines Gegenübers gemacht hat, weil sein Werk eine ebenso treu gewissenhafte wie souveräne Auseinandersetzung mit dem nach Zeit und Charakter so gänzlich Anderen war" (Miller, Komm. in MA 7, S. 775). 
G.s fehlende Autopsie von Werken Cellinis, insbesondere des Perseus in Florenz (vom Salzfass in Wien gibt ihm erst 1815 eine Zeichnung Friedrich Justin Bertuchs (Abb. 68) detailliertere Kunde, die ihn zu einer erläuternden Ergänzung in seinem Anhang veranlasst), ist die ostinate Klage, die seine Übersetzungstätigkeit begleitet. In den Tag- und Jahresheften auf das Jahr 1803 heißt es im Rückblick: "Da ich mich in meinem Leben vor nichts so sehr als vor leeren Worten gehütet, und mir eine Phrase, wobei nichts gedacht oder empfunden war, an andern unerträglich, an mir unmöglich schien, so litt ich bei der Übersetzung des Cellini, wozu durchaus unmittelbare Ansicht gefordert wird, wirkliche Pein. Ich bedauerte herzlich daß ich meine erste Durchreise, meinen zweiten Aufenthalt zu Florenz nicht besser genutzt, mir von der Kunst neuerer Zeit nicht ein eindringlicheres Anschauen verschafft hatte. Freund Meyer, der in den Jahren 1796 und 1797 sich daselbst die gründlichsten Kenntnisse erworben hatte, half mir möglichst aus, doch sehnt' ich mich immer nach dem eigenen, nicht mehr gegönnten Anblick. "Sich allein auf diese von Meyer per Korrespondenz aus Italien übermittelten, zumeist von antimanieristischem Affekt eingefärbten Informationen über Cellinis Werke zu verlassen, scheint G. nicht genügt zu haben. Ganz im Einklang mit der im 18. Jh. dominanten Rezeptionshaltung, die Cellini weniger als einen herausragenden manieristischen bildenden Künstler denn als den Verfasser der Vita und damit als Literaten würdigte, schreibt G. im Anhang, »daß Cellini seinen Nachruhm fast mehr seinen Schriften, als seinen Werken zu verdanken habe« (MA 7, S. 501). Das psychologisch-anthropologische Interesse an Cellini als Mensch scheint bei G. wesentlich stärker ausgeprägt als das an dessen Kunstwerken, die er eher mit antiquarischem Fleiß denn mit Begeisterung im Anhang katalogartig unter dem Titel Hinterlassne Werke aufführt (ebd., S. 497-501).

G.s Interesse an der Person und am Charakter Cellinis ist jedoch kein zufälliges, sondern ein gewissermaßen menschheitsgeschichtlich motiviertes. Programmatisch heißt es am Anfang der Schilderung Cellinis im Anhang: "In einer so regsamen Stadt, zu einer so bedeutenden Zeit, erschien ein Mann, der als Repräsentant seines Jahrhunderts und, vielleicht, als Repräsentant sämtlicher Menschheit gelten dürfte. Solche Naturen können als geistige Flügelmänner angesehen werden, die uns, mit heftigen Äußerungen, dasjenige andeuten, was durchaus, obgleich oft nur mit schwachen unkenntlichen Zügen, in jeden menschlichen Busen eingeschrieben ist. Bestimmter jedoch zeigt er sich als Repräsentanten der Künstlerklasse, durch die Allgemeinheit seines Talents« (ebd., S. 489). Reinhold Schuler hat den G.schen Begriff des »geistigen Flügelmannes « prägnant analysiert: Aus dem militärischen Bereich stammend, bezeichnet er dort eine Art »Vortänzer« einer Reihe, der, meist auffällig groß und damit über seine Kameraden hinausragend, die vom gesamten Glied auszuführenden Bewegungen in besonders prägnanter Weise vormacht. Dies kann nur um den Preis einer gewissen Vereinfachung und Abstraktion geschehen, ohne dass die entsprechenden Bewegungen dadurch das Charakteristische in ihrer wesentlichen Bestimmung verlieren dürfen. Der »Flügelmann « personifiziert damit »die Einfachheit des Gesetzes, aber noch nicht als Formel, sondern als einprägsame Gestalt«; er ist, ganz im G.schen Sinne, das unmittelbare »VorAugenliegen eines Allgemeinen in der besonderen Erscheinung" (Schuler, S. 17). G.s Begriff des "Flügelmannes« belegt somit einmal mehr seinen Hang, im Besonderen das Allgemeine zu suchen, im Individuum die Menschheit zu finden, das punktuelle Ereignis in eine longue durée einzubinden; er ist Metapher für einen morphologischen Blick auf individuelle Manifestationen eines Zeitgeistes, die weniger einmalig werden, weil sie vor allem auf ihren Verallgemeinerungswert hin hinterfragt werden. Flankierend zu diesem Verfahren der Totalisierung setzt G. eine Strategie der Parallelisierung von Lebensläufen in Gang, die es ihm später ermöglichen wird, bioi paralleloi in Cellini und Moses oder auch in Cellini und Filippo Neri zu finden.

G.s Anhang bietet die Schilderung eines Renaissance-Individuums in seinem biographischen, geographischen und künstlerischen Umfeld sowie seiner sozioökonomischen Eingebundenheit in die allgemeine Weltgeschichte: Cellini wird als durch seine Lebensumstände 


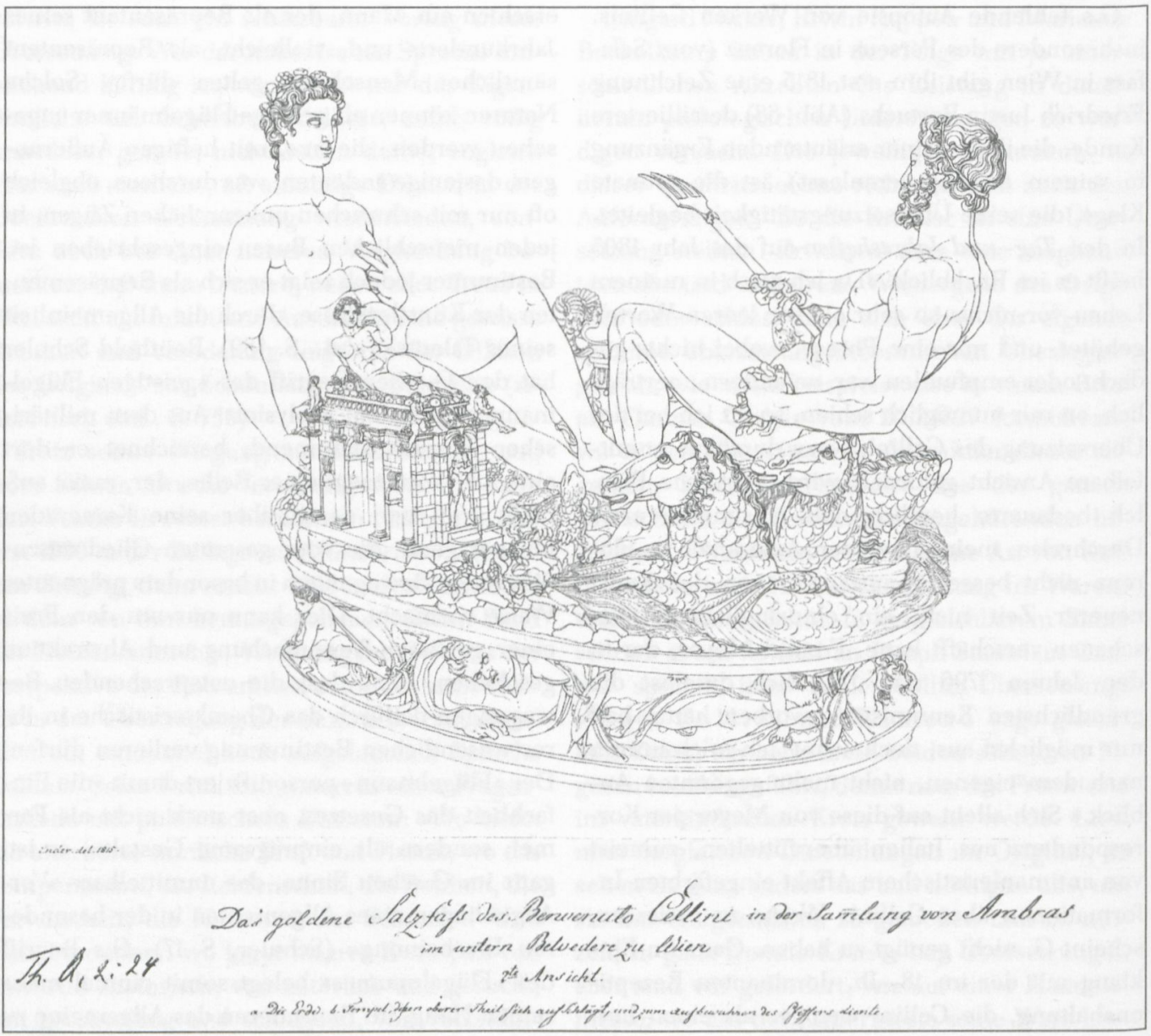

Abb. 68: Friedrich Justin Bertuch: Cellinis `Salzfass«, 1815

maßgeblich geprägt betrachtet; kraft dieser Prägung wird er im Umkehrschluss zu einem herausragenden Repräsentanten seiner Zeit, zu einer exemplarischen Verkörperung des florentinischen Geistes und Charakters des Cinquecento. Nicht von ungefähr trägt der Appendix den sprechenden Titel Anhang zur Lebensbeschreibung des Benvenuto Cellini, bezüglich auf Sitten, Kunst und Technik, der seine sozialhistorische und kulturgeschichtliche Ausrichtung eindeutig markiert. Der Anhang wird so zu einem Dokument für G.s Geschichtsbild: Geschichte ist ihm erkennbar und nacherlebbar im Exemplarischen (Schuler). Personalisierte Geschichtsbetrachtung und Geschichtserzählung hat für G. den Vorteil größerer Lebendigkeit, stärkerer individueller Identifikation mit der Vergangenheit, des prägnanteren Ausdrucks von Zeittypischem. So heißt es in der auf Machiavellis Istorie fiorentine basierenden Flüchtigen Schilderung florentinischer Zustände im Anhang: »Denn, indem man einen merkwürdigen Menschen, als einen Teil eines Ganzen, seiner Zeit, oder seines Geburts- und Wohnorts, betrachtet; so lassen sich gar manche Sonderbarkeiten entziffern, welche sonst ewig ein Rätsel bleiben würden. Daher entsteht, bei jedem Leser, solcher frühern, eignen Lebensbeschreibungen, ein unwiderstehlicher Reiz, von den Umgebungen jener Zeiten nähere Kenntnis $\mathrm{zu}$ erlangen, und es ist ein großes Verdienst leb- 
haft geschriebner Memoiren, daß sie uns, durch ihre zudringliche Einseitigkeit, in das Studium der allgemeinern Geschichte hineinlocken " (MA 7, S. 477). Und in vergleichbarem Sinne hatte G. am 18.4.1796 an Meyer geschrieben: „Die Bearbeitung des Cellini in der ich schon ziemlich weit vorgerückt bin, ist für mich, der ich ohne unmittelbares Anschauen gar nichts begreife, vom größten Nutzen, ich sehe das ganze Jahrhundert viel deutlicher durch die Augen dieses konfusen Individui als im Vortrage des klärsten Geschichtsschreibers."

Indem Cellini für G. zum »Flügelmann« einer ganzen Epoche - der Renaissance - wird, exemplifiziert dieser Ausnahmemensch zugleich in idealer Weise G.s kontinuierliches Interesse an der Interaktion von Einzelschicksal und es umgebender und bedingender Epoche, an »dem Wechselverhältnis von Dichtung und Wahrheit, von Ich und Welt, von Individuum und Geschichte« (Miller, S. 756). So erklärt G. Cellinis Hauptcharakterzug, seine exorbitante Reizbarkeit, wenn sich etwas seinem Willen entgegenstellt, nicht nur als »allgemeine Eigenschaft des Menschencharakters (MA 7, S. 492), sondern darüber hinaus als dem vorherrschend agonalen Zug der Zeit geschuldet, der sich bis in die zeitgleichen kriegerischen Auseinandersetzungen hinein manifestiere, die sämtlich duellhaft gewesen seien. Und Cellini selbst »mit seiner Kunst und mit seinem Lebenswandel « wird ihm zu einem »trefflichen Standpunkt, von dem man, in Absicht auf neue Kunst, vorwärts und rückwärts sehen kann" (an Meyer, 3./9.3.1796). G.s Blick auf Cellini ist der des objektiv-interessierten "Menschenforschers« (Rehm, S. 112), für den der Künstler ein merkwürdiges, also bemerkenswertes Zeitphänomen darstellt: Er ist durch bestimmte, historisch zu rekonstruierende äuBere Umstände zu dem geworden, was er in seiner Zeit repräsentierte. Dadurch erhält Cellinis Vita für G. den Rang eines exemplarischen Lebens, eines Exemplums für die Menschheit, und dies in doppelter Hinsicht: "nach innen durch das Undeterminierte, Bildsame der Individualität, die ein Beispiel des Menschlichen von schier unerschöpflichem Reichtum und imponierender Vielseitigkeit ermöglicht, und nach außen durch den im Individuum und durch das
Individuum greifbaren Weltgehalt« (Schuler, S. 53).

G.s Charakterschilderung Cellinis im Anhang lässt nicht auf übertriebene Sympathie für diese Art von egomanisch-überbordender Subjektivität schließen: Für G. ist Cellini ein schnell produzierender Virtuoso, der einen Hang ins Ornamental-Gefällige hat, sich durch mangelnde Tiefe des Denkens und generelle Oberflächlichkeit auszeichnet und sich künstlerisch nicht hinlänglich an der Antike orientiert. In G.s Augen ist er übermäßig leidenschaftlich, selbstgefällig, eigensinnig und ungeduldig, ein wenig wählerischer Frauenheld und Erotomane (die homoerotische Komponente von Cellinis Sinnlichkeit spart $\mathrm{G}$. bezeichnenderweise aus bzw. sublimiert sie zur Bewunderung für jungmännliche Körperschönheit, so wie er auch in seiner Übersetzung grundsätzlich die skatologische oder sexualisierte Ausdrucksweise des Künstlers abmildert). Cellinis sämtliches geistiges und seelisches Vermögen befindet sich in einem Zustand andauernder Unausgeglichenheit: »Dieser leidenschaftlich-selbstische Mann « (MA 7, S. 495) ist ein gewaltsames und ungebändigtes Naturwesen, ein "gleichsam unbegrenztes Individuum « (ebd., S. 502) mit einer »leidenschaftlichen, nur aufs Gegenwärtige gerichteten Natur« (ebd., S. 504). Er ist der Prototyp selbstbewusster und rücksichtslos-egomaner Subjektivität, die nicht erst für Jacob Burckhardt in seiner Cultur der Renaissance in Italien (1860) zum bedrohlichen Signum von Modernität wurde, indem er Cellini als einen Menschen charakterisierte, »der Alles kann, Alles wagt und sein $\mathrm{Ma}$ in sich selber trägt« (Burckhardt, S. 333). Sein misanthropischkulturkritisches Fazit lautete: »Ob wir es gerne hören oder nicht, es lebt in dieser Gestalt ein ganz kenntliches Urbild des modernen Menschen" (ebd.).

Cellinis Charakter färbt in G.s Wahrnehmung auf sein künstlerisches Schaffen ab und prägt seine ästhetischen Vorstellungen. Was Meyer ihm in despektierlichem Ton über Cellinis Manier aus Italien zu berichten weiß, spiegelt für G. im Umkehrschluss klar den Charakter des Künstlers - eine Form von Biographismus, die insbesondere bei mangelnder eigener Anschauung der Kunstwerke methodisch zweifelhaft ist 
und die Kehrseite von G.s Postulat der Einheit von Kunst und Leben darstellt. So schreibt er am 22.7.1796 an Meyer zurück nach Florenz: "Das, was Sie von seinen Arbeiten sagen, trifft mit seinem Charakter und seinem Schicksal vollkommen überein; seine Bildung ging vom Einzelnen aus, und bei seiner großen, puren Sinnlichkeit wäre es ein Wunder gewesen, wenn er sich durch Reflexion hätte zum Ganzen erheben sollen. « Das aber ist für G. der Imperativ jedes gelungenen Lebens und jedes gelungenen Kunstwerks: Abbild einer gerundeten, ganzheitlichen Bildung zu sein. Damit weicht Cellini als Künstler und Mensch deutlich von G.s Idealvorstellungen $\mathrm{ab}$ - in aestheticis besonders im Hinblick auf eine wild vagierende und ungebändigt wirkende Einbildungskraft, die durch mangelnde Reflexion auf das eigene Handeln und Denken, durch eine dem Künstler eigene Naivität bedingt ist.

Cellinis Visionen sind für G. Ausgeburten dieser überschießenden Einbildungskraft, die sich in Krisensituationen verhinderter künstlerischer Aktion kompensatorisch manifestiert, indem sie dem Verstand Bilder vorgaukelt. Cellinis religiöse Bedürfnisse schließlich sind ebenfalls Ausfluss psychischer Kompensation, in der die »menschliche Natur« bestrebt ist, die Einseitigkeiten und Unausgeglichenheiten des KünstlerCharakters gleichsam wieder ins harmonische Gleichgewicht aller Körper- und Geisteskräfte zu bringen: „Wie aber die menschliche Natur sich immer ganz herzustellen und darzustellen genötigt ist, so erscheint, in diesen wüsten, sinnlichen Welträumen, an unserem Helden, so wie an seinen Umgebungen, ein sittliches und religioses Streben, das erste im größten Widerspruch mit der leidenschaftlichen Natur, das andere, zu Beruhigung, in verdienten und unverdienten, unausweichlichen Leiden « (MA 7, S. 493).

Cellini war G. weder stypmäßig« noch ästhetisch geheuer, wie eine Äußerung in einem Brief an Meyer vom 30.10.1796 belegt: "Da ich eben in meinem Cellini an den Guß seines Perseus komme, und durch Sie von seinen herrlichen Vorgängern höre, so wird es mir recht deutlich wie man von dem reinen Wege der Natur und der gefühlten und überlegten Kunst, durch
Phantasie und Leidenschaft bey einem angebohrnen großen Talent, auf den Weg der Phantasterey und Manier gerathen könne und müsse. Wenn man hört, wie er gearbeitet hat, und was er an sich rühmt, so ahndet man was seine Werke seyn müssen.« Der Manierismus als antimimetischer Stil und Ausdruck überbordender künstlerischer Subjektivität musste G.s klassizistisches Ideal der Mäßigung und Beruhigung irritieren. Der potentiell bedrohlichen Modernität von Cellinis Werken werden daher im Anschluss an diese Briefstelle die »Kleinodien « der Florentiner Skulptur und Malerei des Quattrocento entgegengestellt. Klaus Herding sieht diese Dialektik von Nähe und Distanz, von Faszination und Abstoßung bis hin in die Sprache der G.schen Übersetzung am Werke: Indem G. einen bewusst altertümelnden Duktus in seinem Text verwende, werde ihm die Sprache selbst zum »Mittel der Distanzierung Cellinis« (Herding, S. 381).

Als Repräsentant des Renaissancekünstlers vertritt Cellini für G. nur eine Seite des typisch Florentinischen: den fortwährenden Konflikt; das Streitbare, Agonale; das Unausgeglichene. Dagegen steht - gewissermaßen als Antidotum - das bürgerliche Fundament der Florentiner Civitas, eine Form konservativer Wohlanständigkeit und emsigen Gewerbefleißes: „Erwerben, Erhalten, Erweitern, Mitteilen, Genießen, gehen gleichen Schrittes und in diesem lebendigen Ebenmaß läßt uns die bürgerliche Weisheit ihre schönsten Wirkungen sehen" (MA 7, S. 483). Wie Angelika Jacobs zeigen konnte, hat der Anhang für G. auch die Funktion, diesen ihm so suspekten Künstlertypus durch seine Einbindung in den zeitgeschichtlichen Kontext zu zügeln, zu bändigen und zu ^normalisieren<, seine exorbitante Individualität aus den äußeren Umständen heraus zu erklären und damit in ihrer Einmaligkeit zu relativieren: »Der eklatante Abstand zwischen Cellinis und Goethes Kunstanschauung motiviert die Abfassung des fragmentarischen historischen Kommentars. In diesem Sinne trägt die Beschäftigung mit der Cellini-Vita ex negativo zur Konturierung der klassizistischen Kunstdoktrin bei, so wie sie den Weg in die Konzeption des eigenen autobiographischen Vorhabens und in die historiographi- 
sche Darstellung des Cinquecento weist. Die Erfahrung der Fremdartigkeit dieser Autobiographie schärft Goethes Aufmerksamkeit in Bezug auf die Modernität des eigenen Kunstbegriffs und der eigenen Auffassung von Subjektivität« (Jacobs, S. 263). Dass der Anhang Fragment blieb, mag darauf hindeuten, dass der Versuch der anverwandelnden Dämpfung von Cellinis regel- und grenzüberschreitender Individualität nicht gänzlich gelungen ist.

Für Jacobs ist G. zur Zeit der Cellini-Übersetzung auf der Suche "nach einem modernen Ichprinzip«, "nach frühen Formen künstlerischen Selbstbewusstseins, die er in Cellinis Vit a zwar vorfindet, die aber nicht den Erwartungen entsprechen und in ihrer Fremdheit die historische Einordnung und Kommentierung erforderlich machen" (ebd., S. 255). So scheint G.s vergleichsweise ausführliches Referat von Cellinis Traktat über die Goldschmiedekunst die Absicht zu verfolgen, den sich zum Bildhauer Autonomisierenden wieder auf den Boden des Handwerks zurückzuholen, von dem er gerade mit allen Kräften wegstrebt. Die »Fähigkeit zu allem mechanischen " (MA 7, S. 489), die G. als Cellinis herausragendes Talent betont, erdet damit gewissermaßen die überschießende und ungezügelte Einbildungskraft des Virtuosen. Und die Einordnung des selbsternannten Ausnahmekünstlers, der alle Vorbilder überbietet und keine Grenzen kennt, in eine Art Rubrikenschema von Vorläufern und Zeitgenossen und deren überragenden künstlerischen Hervorbringungen dient ebenfalls dem Zwecke der Relativierung und Kontextualisierung und damit der Verallgemeinerung. Selbst Cellinis professionelle Emanzipation vom Kunsthandwerk zur freien Kunst wird mit dem Hinweis auf Donatello, Filippo Brunelleschi und Lorenzo Ghiberti - "sämtlich zuerst Goldschmiede" (G. an Meyer, 8.2.1796) - in ihrer Einmaligkeit relativiert. Doch trotz der konstatierten starken typmäßigen Differenzen zu seinem "widerspenstigen Doppelgänger« (Miller, S. 766) befasste sich G. jahrelang mit Cellinis Text, was auf eine hinlängliche Faszination schließen lässt. Hierbei mag ihn u.a. die Frage nach dem Funktionieren oder Scheitern von Patronagebeziehungen interessiert haben, wie Cellini sie in Frankreich oder Florenz erlebte; eine Frage, die nicht nur eine Reminiszenz an das Tasso-Thema darstellte, sondern auch in möglicher Parallelität zu G.s eigenen Erfahrungen am Weimarer Hof stand.

Der von der Forschung immer wieder betonte hauptsächliche Identifikationspunkt mit dem Cellini-Thema scheint jedoch darin gelegen $\mathrm{zu}$ haben, dass die Übersetzung von Cellinis Vita G. ein Modell für das eigene autobiographische Schreiben lieferte. Cellini wird für G. u. a. zum Kronzeugen für den richtigen Zeitpunkt autobiographischer Reflexion, wenn er ihn 1823 zustimmend in einem skizzenhaften Text in Über Kunst und Altertum zitiert: "Cellini sagt, wenn ein Mann, der glaubt, etwas geleistet und ein bedeutendes Leben geführt zu haben, im vierzigsten Jahre steht, so soll er seine Lebensbeschreibung beginnen, die ereignisvolle Zeit seiner Jugend treulich aufzeichnen und in der Folge weiter fortfahren. Cellini hat ganz Recht: denn es ist keine Frage, daß uns die Fülle der Erinnerung, womit wir jene ersten Zeiten zu betrachten haben, nach und nach erlischt, daß die anmutige Sinnlichkeit verschwindet und ein gebildeter Verstand durch seine Deutlichkeit jene Anmut nicht ersetzen kann« (MA 14, S. 572). Dass die Sicherung des Nachruhms nicht allein den eigenen Werken zu überlassen sei, sondern aktiv durch autobiographisches Schreiben unterstützt werden müsse, wenn man das eigene Leben zu einem gelungenen und damit vor Gott und der Welt legitimierten stilisieren will, war eine Lektion, die G. ebenfalls von Cellini lernen konnte. Und bereits im ersten Schema zu Dichtung und Wahrheit hatte G. die Vorbildhaftigkeit von Cellinis Vita für sein eigenes autobiographisches Schreiben konzediert.

Norbert Miller hat darauf hingewiesen, dass das späte Erscheinungsdatum der Gesamtübersetzung im Jahr 1803 diese in den Kontext einer ganzen Reihe von biographischen und autobiographischen Schreibversuchen G.s stelle. Er spricht hierbei - in terminologischer Anlehnung an Theodor Gottlieb von Hippel - von »stellvertretenden Lebensläufen in aufsteigender Linie* (Miller, S. 765), an deren Anfang Cellini steht, dem dann die Lebensbeschreibungen Johann Joachim Winckelmanns und Jakob Philipp Hackerts folgen, und an deren Ende sich G. selbst 
stellt. Diese Biographien scheinen G. als >Probeläufe für die eigene »Selberlebensbeschreibung « (Miller, S. 770) gedient zu haben, wie man seiner Äußerung im Vorwort zu Dichtung und Wahrheit entnehmen kann, der an dieser prominenten Stelle die Rolle der Selbstversicherung über das Monumentalprojekt autobiographischen Schreibens zukommt: »Denn dieses scheint die Hauptaufgabe der Biographie zu sein, den Menschen in seinen Zeitverhältnissen darzustellen, und zu zeigen, in wiefern $\mathrm{ihm}$ das Ganze widerstrebt, in wiefern es ihn begünstigt, wie er sich eine Welt- und Menschenansicht daraus gebildet, und wie er sie, wenn er Künstler, Dichter, Schriftsteller ist, wieder nach außen abspiegelt. Hiezu wird aber ein kaum Erreichbares gefordert, daß nämlich das Individuum sich und sein Jahrhundert kenne, sich, in wiefern es unter allen Umständen dasselbe geblieben, das Jahrhundert, als welches sowohl den willigen als unwilligen mit sich fortreißt, bestimmt und bildet, dergestalt daß man wohl sagen kann, ein Jeder, nur zehn Jahre früher oder später geboren, dürfte, was seine eigene Bildung und die Wirkung nach außen betrifft, ein ganz anderer geworden sein « (MA 16, S. 11).

\section{Literatur:}

Bockelkamp, Marianne: Goethes Cellini-Übersetzung. Diss. Freiburg/Br. 1960. - Burckhardt, Jacob: Die Cultur der Renaissance in Italien. Ein Versuch.
Basel 1860. - Cellini, Benvenuto: Vita. Testo critico. Hg. von Orazio Bacci. Florenz 1901. - Dewitz, Komm. in FA I, 11, S. 821-947. - Fischer, Richard: ,Ein Repräsentant seines Jahrhunderts<. A Portrait of the Artist in Goethe's >Anhang s to the Autobiography of Benvenuto Cellini. In: Michigan Germanic Studies 14 (1988), S. 85-106. - GMB. - Hausmann, Frank-Rutger: Benvenuto Cellini. In: GHB 3, S. 500-506. - Herding, Klaus: >Allegro, deciso, con impetor. Cellini als Wunschbild des Künstlers seit Goethe. In: Nova, Alessandro/Schreurs, Anna (Hg.): Benvenuto Cellini. Kunst und Kunsttheorie im 16. Jahrhundert. Köln u.a. 2003, S. 380-413. Jacobs, Angelika: Goethe und die Renaissance. Studien zum Konnex von historischem Bewußtsein und ästhetischer Identitätskonstruktion. München 1997. - Koppen, Erwin: Goethes Benvenuto Cellini. Glanz und Elend einer Übersetzung. In: GJbWien 81-83 (1977-1979), S. 247-262. - Koranyi, Stephan: Autobiographik und Wissenschaft im Denken Goethes. Bonn 1984. - Löhneysen, Wolfgang von: Cellini. In: Zastrau, Alfred (Hg.): Goethe-Handbuch. Goethe, seine Welt und seine Zeit in Werk und Wirkung. Bd. 1. Stuttgart 1961, Sp. 1589-1604. Miller, Komm. in MA 7, S. 721-993. - Mommsen, Momme: Die Entstehung von Goethes Werken in Dokumenten. Bd. 2. Berlin 1958. - Nickel, Irmgard: Goethes Übersetzung der Vita des Benvenuto Cellini. In: GJb 81 (1964), S. 223-228. - Rehm, Walther: Das Werden des Renaissancebildes in der deutschen Dichtung vom Rationalismus bis zum Realismus. München 1924. - Schuler, Reinhard: Das Exemplarische bei Goethe. Die biographische Skizze zwischen 1803 und 1809. München 1973. Vossler, Karl: Einleitung des Herausgebers. In: Goethe, Johann Wolfgang: Werke. Hg. von Karl Heinemann. Bd. 27. Leipzig, Wien [1903], S. 7-14. Christine Tauber 